\title{
Visual Morbidity in Diabetic Retinopathy Associated with Diabetic Nephropathy - Our Experience in a Multispecialty Tertiary Hospital in Chennai, India
}

\author{
Praveena Venkatakrishnan ${ }^{1}$, Radha Annamalai², Muthayya Muthukumar ${ }^{3}$ \\ 1, 2,3 Department of Ophthalmology, Sri Ramachandra Institute of \\ Higher Education and Research, Chennai, India.
}

\section{ABSTRACT}

\section{BACKGROUND}

The increased prevalence of diabetes mellitus (DM) worldwide has resulted in worsening diabetic retinopathy (DR) and nephropathy. The pathophysiological changes that occur at a cellular and anatomical level are similar in DR and DN. The risk factors for DR and DN are common hence investigating both is paramount to reduce morbidity. We wanted to study the association of diabetic retinopathy and diabetic nephropathy, their ophthalmic features, patterns of vision loss and extent of ocular morbidity.

\section{METHODS}

This is a retrospective observational study performed on 100 diabetic patients over 2 years. Patients with a confirmed diagnosis of DN based on clinical evaluation and laboratory tests were included. Ophthalmic evaluation and investigations were done and DR was classified based on early treatment diabetic retinopathy study (ETDRS).

\section{RESULTS}

Mild non-proliferative diabetic retinopathy (NPDR) occurred in $60 \%$, moderate to severe NPDR in $9 \%$, proliferative diabetic retinopathy (PDR) in $4 \%$ and no diabetic retinopathy in $27 \%$. Macula was involved in $48 \%$ with clinically significant macular edema (CSME) in $33 \%$ and ischemic maculopathy in $5 \%$. Retinopathy occurred in 3 $\%$ after 5 years and in $40 \%$ after 20 years of DM. The incidence of DN +DR was $65 \%$ and statistical significance was noted with longer duration of diabetes, higher serum creatinine, proteinuria, lower haemoglobin, decreased GFR, higher age and higher lipid levels $(\mathrm{P}=0.04)$. Improvement in vision was seen in $63 \%$ after blood sugar control with laser photocoagulation, $27 \%$ with laser alone and $7 \%$ of patients with intravitreal anti-VEGF

\section{CONCLUSIONS}

DR and DN have an overlapping significant association and all patients have to be screened for both to prevent ocular morbidity.

\section{KEY WORDS}

Diabetic Retinopathy, Diabetic Nephropathy, Vision, Glomerular Filtration Rate, Macular Edema.
Corresponding Author:

Dr. Radha Annamalai,

Plot 1152, 35th Street, 18th Main

Road, Anna Nagar West,

Chennai - 600040,

Tamil Nadu, India.

E-mail: drradhasrmc@gmail.com

DOI: $10.14260 /$ jemds/2021/589

How to Cite This Article:

Venkatakrishnan $P, \quad$ Annamalai $R$, Muthukumar M. Visual morbidity in diabetic retinopathy associated with diabetic nephropathy - our experience in a multispecialty tertiary hospital in Chennai, India. $J$ Evolution Med Dent Sci 2021;10(34):2888-2893, DOI: $10.14260 /$ jemds $/ 2021 / 589$

Submission 16-04-2021,

Peer Review 19-06-2021,

Acceptance 26-06-2021,

Published 23-08-2021.

Copyright (C) 2021 Praveena Venkatakrishnan et al. This is an open access article distributed under Creative Commons Attribution License [Attribution 4.0 International (CC BY 4.0)] 


\section{BACKGROUND}

The increased prevalence of diabetes mellitus (DM) worldwide has resulted in worsening diabetic retinopathy (DR) and nephropathy. ${ }^{1}$ The similarity of features in diabetic retinopathy and nephropathy is due to the resemblance of microvasculature. ${ }^{2}$ The duration of diabetes, glycaemic control, elevated blood pressure, lipid profiles, serum $\mathrm{HbA1c}$, change in retinal vessel calibre and genetic factors contribute to the progression of DR. ${ }^{3}$ Diabetic nephropathy (DN), is an important precursor to the development of chronic kidney disease. ${ }^{4} \mathrm{DN}$ is characterized by a reduction of glomerular filtration rate (GFR) and albuminuria. ${ }^{5}$ The presence of albuminuria signifies the presence of vasculopathy and endothelial abnormalities that worsen morbidity. ${ }^{6}$

As in the case of DR, the major risk factors identified for DN include prolonged duration of diabetes, poor glycaemic control, and hypertension. Patients with severe diabetic nephropathy sometimes present with proliferative retinopathy, persistent macular oedema or / and haemorrhagic complications including neovascularization and vitreous haemorrhage. ${ }^{7}$ Optimizing blood-sugar together with tight control of blood pressure can reduce the risk of developing both DR and DN. These were evaluated in the prospective diabetes study done in the United Kingdom prospective diabetes study (UKPDS) trial. ${ }^{8}$

Chronic hyperglycaemia leads to basement membrane thickening causing loss of pericytes, endothelial cell proliferation and an alteration in blood-retinal barrier. Aggregation of platelets and vascular occlusion lead to hypoxia of the retina and advancement of diabetic retinopathy changes. The disarrangement of microstructure and dysfunction of microcirculation lead to vascular hyperpermeability and microaneurysm formation. Excessive vascular leakage with resultant loss of proteins and lipids from the vessel wall leads to the accumulation of fluid in the inner layers of the retina resulting in diabetic macular oedema. ${ }^{9}$

The newer inflammatory theory which puts vascular endothelial growth factors (VEGF) in the centre of the aetiology of diabetic macular oedema and retinopathy says that the accumulation of advanced glycated end-products (AGEs) leads to the inflammation and release of mediators such as intracellular adhesion molecules (ICAM) and vascular cell adhesion protein (V - CAM) which in turn increase vascular permeability and cause progression of retinopathy and macular oedema. ${ }^{10}$

The purpose was to study the association of diabetic retinopathy and diabetic nephropathy, their ophthalmic features, patterns of vision loss and extent of ocular morbidity.

\section{METHODS}

This is a retrospective observational study from Sri Ramachandra Institute of Higher Education and Research, Chennai, that was performed on 100 patients for 2 years from June 2018 to April 2020 at a tertiary care eye centre in India. An ethics committee approval was obtained (CSP - MED / 21 / MAR / 67 / 47). All patients with a confirmed diagnosis of DN based on the American diabetes association guidelines for micro and macroalbuminuria ${ }^{11}$ were included in the study, the features of which are depicted in table 1. Clinical evaluation and laboratory tests such as blood sugars, HbA1c, fasting lipid profile, complete blood count, renal function test, urine analysis to look for protein (specifically albumin and globulin), red blood cells, specific gravity and signs of previous infections were noted. We also measured serum uric acid, blood urea nitrogen, serum creatinine and serum electrolytes (potassium, sodium and magnesium). Glomerular filtration rate was calculated for all patients with DN using bodyweight and their creatinine values, according to Cock Croft Equation. ${ }^{12}$

GFR $=140-$ AGE in yrs. $\times$ WEIGHT in kg $/ 72 \times$ CREATININE in $\mathrm{mg} / \mathrm{dl}$. Patients with advanced kidney disease were subjected to a renal biopsy to look for microvascular complications and study the histopathological changes related to diabetes.

All type 1 and type 2 diabetes mellitus patients inclusive of both new and old were incorporated in the study. Patients with pre-existing comorbidities were excluded from the study. A detailed history was obtained with regard to treatment, the drugs used with dosage and duration.

Ophthalmic evaluation consisted of slit-lamp examination, indirect ophthalmoscopy, tonometry, and refraction. Ancillary ophthalmic investigations including colour vision, Amsler's grid, perimetry, fundus fluorescein angiography, optical coherence tomography and B scan ultrasonography were performed during the initial and follow up visits.

Patients were reviewed every month to look for a response to treatment and to detect all ocular complications. All patients had a follow up of at least one year. During active disease, weekly reviews were done. As part of monitoring, complete blood counts, renal function tests and blood sugars were done every 3 months. Retinopathy changes were classified into nonproliferative and proliferative diabetic retinopathy. The nonproliferative variant was divided into mild, moderate and severe using ETDRS classification and the presence of clinically significant macular oedema was noted..$^{13}$

\begin{tabular}{|c|c|c|}
\hline Stages & $\begin{array}{l}\text { Albuminuria Cut-Off } \\
\text { Values (Ref. 14) }\end{array}$ & Clinical Characteristics (Ref. No.) \\
\hline \multirow{7}{*}{$\begin{array}{l}\text { Micro- } \\
\text { albuminuria }\end{array}$} & $20-199 \mu \mathrm{g} / \mathrm{min}$ & $\begin{array}{l}\text { Abnormal nocturnal decrease of blood } \\
\text { pressure and increased blood pressure } \\
\text { levels (163) }\end{array}$ \\
\hline & $30-299 \mathrm{mg} / 24 \mathrm{~h}$ & $\begin{array}{l}\text { Increased triglycerides, total and LDL } \\
\text { cholesterol, and saturated fatty acids } \\
(164,165)\end{array}$ \\
\hline & \multirow{5}{*}{$30-299 \mathrm{mg} / \mathrm{g}^{*}$} & $\begin{array}{l}\text { Increased frequency of metabolic } \\
\text { syndrome components (166) }\end{array}$ \\
\hline & & Endothelial dysfunction (167) \\
\hline & & $\begin{array}{l}\text { Association with diabetic retinopathy, } \\
\text { amputation, and cardiovascular disease } \\
\text { (168) }\end{array}$ \\
\hline & & $\begin{array}{c}\text { Increased cardiovascular mortality (2, } \\
169)\end{array}$ \\
\hline & & Stable GFR (82) \\
\hline \multirow{4}{*}{$\begin{array}{l}\text { Macroalbuminu } \\
\text { ria† }\end{array}$} & $\geq 200 \mu \mathrm{g} / \mathrm{min}$ & Hypertension (99) \\
\hline & $\geq 300 \mathrm{mg} / 24 \mathrm{~h}$ & $\begin{array}{c}\text { Increased triglycerides and total and LDL } \\
\text { cholesterol (170) }\end{array}$ \\
\hline & \multirow[t]{2}{*}{$\geq 300 \mathrm{mg} / \mathrm{g}$} & $\begin{array}{c}\text { Asymptomatic myocardial ischemia (171, } \\
172)\end{array}$ \\
\hline & & Progressive GFR decline $(83,84)$ \\
\hline$T a b$ & hetic Nonh & $\begin{array}{l}\text { y Stages: Cut-Off Values of } \\
\text { Main Clinical Characteristics }\end{array}$ \\
\hline
\end{tabular}

\section{Statistical Analysis}

Sample size calculation: Sample size was calculated keeping a study done by Samreen Jamal et al. to analyse the "Frequency and grading of diabetic retinopathy in diabetic end-stage renal disease patients". ${ }^{14}$ Keeping an alpha error of $5 \%$ and a CI of 
$95 \%$, the sample size was calculated as 96 which we rounded off to 100 .

Analysis - All collected data were analysed using IBM SPSS statistics software, version 23.0. The frequency analysis for distribution of data and percentage analysis was done for categorical variables. Mean and standard deviation was analysed using continuous variables. Kappa coefficient was used for correlating test values. Statistical significance of categorical data was done using chi-square test and a probability value of $\mathrm{P}<0.05$ was considered as statistically significant.

\section{RESULTS}

This was a retrospective study performed on 100 patients. We had $16 \%$ patients with type 1 and $84 \%$ with type 2 DM. No features of diabetic retinopathy were seen in $27 \%$, mild nonproliferative diabetic retinopathy in $60 \%$, moderate to severe non-proliferative diabetic retinopathy (NPDR) in $9 \%$, and proliferative diabetic retinopathy (PDR) in $4 \%$ of the cohort. This group was further divided into those with isolated DR group which accounted for $35 \%$ and the DR with DN in association accounted for $65 \%$.

In the set with combined retinopathy and nephropathy, mild NPDR occurred in $29 \%$, moderate NPDR in $15 \%$, severe NPDR in $9 \%$, very severe NPDR in $5 \%$ and PDR in $7 \%$. There was a definite increase in the prevalence of retinopathy in those with nephropathy. Involvement of the macula was present in $48 \%$ of patients.

Clinically significant macular oedema was noted in $33 \%$ and ischemic maculopathy in $5 \%$. Retinopathy was related to the presence of associated blood pressure, serum creatinine, and glomerular filtration rate and serum potassium levels. The prevalence and severity of retinopathy were associated with a longer duration of diabetes. Incidence of retinopathy with regard to duration occurred in $3 \%$ after 5 years of DM and in $40 \%$ after 20 years.

Among these 100 patients, 60 were males and 40 were females. A renal biopsy had been performed in 21 patients and the findings were predominantly interstitial inflammation with cellular infiltration in 15 of those patients. The mean GFR was $57 \pm 33 \mathrm{~mL} / \min / 1.73 \mathrm{~m}^{2}$, and proteinuria level was $4.21 \mathrm{~g}$ / day (range, 0.03-24.00 g / day).

The isolated DR group accounted for $35 \%$ and the DR with DN accounted for $65 \%$. Compared with the DN group, patients in DN + DR group were more likely to have a longer duration of DM, higher levels of serum creatinine and proteinuria, lower levels of haemoglobin, increased incidence of haematuria, and decreased GFR than participants without DR. This was found to be statistically significant with $\mathrm{P}=0.04$. There was a considerable effect of age, incidence of hypertension, blood urea nitrogen (BUN), obesity, blood sugar levels (FBS, PPBS and HbA1c), uric acid, lipid profile (highdensity lipoprotein, low-density lipoprotein, triglyceride, total cholesterol in those who developed DR in DN. Another significant risk factor that was identified in association with DR was haematuria which was seen in $12 \%$ of patients. $21 \%$ of patients with renal failure had no evidence of retinopathy.

An improvement in vision was noted in $63 \%$ following control of blood sugar combined with laser photocoagulation,
$27 \%$ with laser photocoagulation alone and in $7 \%$ of patients with intravitreal anti-vascular endothelial growth factor.

\begin{tabular}{|ccc|}
\hline Severity of Retinopathy & Number of Participants (N) & Percentage \% \\
Mild & 44 & 60 \\
Venous dilatation & 26 & 36 \\
Microaneurysms & 20 & 27 \\
Hard exudates & 15 & 21 \\
Moderate to severe & 7 & 9 \\
Cotton wool spots & 7 & 9 \\
IRMA & 5 & 6 \\
Proliferative diabetic & 3 & 4 \\
retinopathy & 1 & 1 \\
NVD & 2 & 3 \\
NVE & 2 & \\
\hline Table 2. No. of Participants in Which Various Clinical Signs of \\
Retinopathy Were Seen in Our Study Population \\
\hline
\end{tabular}

\begin{tabular}{|ccc|}
\hline Classification of Patients & $\begin{array}{c}\text { Number of Participants } \\
\text { (N) }\end{array}$ & Percentage \% \\
Retinopathy only & 26 & 35 \\
$\begin{array}{c}\text { Nephropathy with } \\
\text { retinopathy }\end{array}$ & 47 & 65 \\
\hline Table 3. No. of Participants with Retinopathy and Nephropathy \\
\hline
\end{tabular}

\begin{tabular}{|ccc|}
\hline Grade of Retinopathy & No of Participants (N) & Percentage \\
Mild NPDR & 14 & $29 \%$ \\
Moderate NPDR & 7 & $15 \%$ \\
Severe NPDR & 4 & $9 \%$ \\
Very Severe NPDR & 2 & $5 \%$ \\
PDR & 3 & $7 \%$ \\
\hline Table 4. Association of Diabetic Retinopathy with Nephropathy \\
\hline N = All patients with diabetic retinopathy and nephropathy \\
\hline
\end{tabular}

\section{DISCUSSION}

DM affects about 463 million people worldwide. Diabetes currently affects more than 62 million Indians, which is more than $7.2 \%$ of the adult population. ${ }^{15} \mathrm{~A}$ single pathway due to microvascular damage appears to be the cause of renal and retinal involvement in DM. This link between both organs getting involved as a consequence of microvascular disease can have varying consequences on vision and renal function both of which can affect the quality of life. They coexist or may occur in isolation and need evaluation to be performed to prevent life-threatening complications.

Patients with diabetes mellitus and diabetic retinopathy (DR) are prone to develop diabetic nephropathy (DN) and the incidence of retinopathy is higher in those with DN. In this study, we aimed to clarify the relationship between DR and the progression of DN in these patients. Our findings indicated that the severity of glomerular lesions was significantly associated with $\mathrm{DR}^{16}$ and DR and was an independent risk factor for the renal outcomes in patients with DN. An exact incidence on which precedes the other is difficult to deduce as it varies based on other systemic influences. However, a definite correlation of severity and progression suggesting that DR may predict the renal prognosis of patients in DN and vice versa was established on our study patients.

An estimation of the presence and severity of retinopathy is usually made based on direct ophthalmic evaluation of anatomical changes combined with an evaluation of visual acuity with supportive evidence from fundus fluorescein angiography [FFA] and optical coherence tomography [OCT]. Nephropathy unlike retinopathy is defined using functional abnormalities such as GFR, proteinuria, haematuria and microalbuminuria.

Sabanayagam et al. demonstrated that CKD is usually associated with diabetic retinopathy only when albuminuria is 
present and this demonstrates the need for albuminuria to be an associated feature of CKD. 17

Kimmelstiel and Wilson in 1936 described a clinicopathological syndrome characterized by hypertension, renal failure with albuminuria, widespread oedema and retinitis in longstanding and often mild diabetics whose kidneys demonstrated histologically what they termed intercapillary glomerulosclerosis. ${ }^{18}$ This disease is extensively associated with nephrotic syndrome and long-standing diabetes.

This requires a renal biopsy for confirmation. Many of our patients underwent renal biopsy to look for interstitial changes in the kidney that could have probably led to the microvascular changes that have occurred at the cellular level. Many patients with ischemic nephropathy and Kimmelstiel Wilson disease commonly present with cotton wool spots and arteriolar narrowing with retinal exudates which was a consistent retinal feature in many of the patients in our study. It is however essential to distinguish this from ischemic nephropathy and hypertensive retinopathy. The role of free fatty acids (FFA) to assess the ischemic changes in DR becomes essential and capillary dropouts and foveal avascular zone (FAZ) expansion are the key findings in ischemic retinopathy. A strong suspicion of nephropathy must be made in all patients presenting with retinopathy as suggested by Lee et al. ${ }^{19}$ demonstrated that ischemic DR characterized with extensive capillary nonperfusion is a possible prognostic factor for the progression of CKD and a strong index of suspicion must be maintained in patients who present with profound visual loss that is out of proportion with retinal findings which is strongly suggestive of ischemic maculopathy.

However, this relationship between anatomical changes causing retinopathy and functional changes in nephropathy in DM has remained inconclusive. Studies have also reported the incidence of both features in isolation. Advanced renal changes have occurred in the absence of retinopathy. ${ }^{20}$

We found that there was a significant increase in the incidence and progression of retinopathy in patients with DN. The severity of retinopathy increased with the increasing severity of nephropathy. Diabetic maculopathy also correlates with DN. Biswas et al. studied diabetic maculopathy and diabetic nephropathy in 100 patients in the Indian population and found a strong association between the two. ${ }^{21}$

The greatest association was found between DM, hypertension and increased lipid profile (triglycerides, LDL, cholesterol) with all three playing a role in the severity of retinopathy and nephropathy. ${ }^{22}$ In a study by JL Wilkinson et al. they established these risk factors and found a strong correlation between vision loss, CKD and these risk factors with increasing prevalence of PDR in patients with advanced CKD. ${ }^{23}$ Our results are also similar to a study done by Ahmed $\mathrm{M}$ et al. in the Sudanese population and these results show us that hypertension and hyperlipidaemia are important risk factors for nephropathy and retinopathy across population demographics. ${ }^{24}$ Among laboratory tests for DM, HbA1c seemed to have a bigger role in the determination and assessment of the visual status. Clinically significant macular oedema [CSME] was associated with higher LDL, total cholesterol values, HbA1c levels and raised serum creatinine. The decreased prevalence of macular oedema is probably related to modifications of osmotic pressures that occur during dialysis. ${ }^{25}$

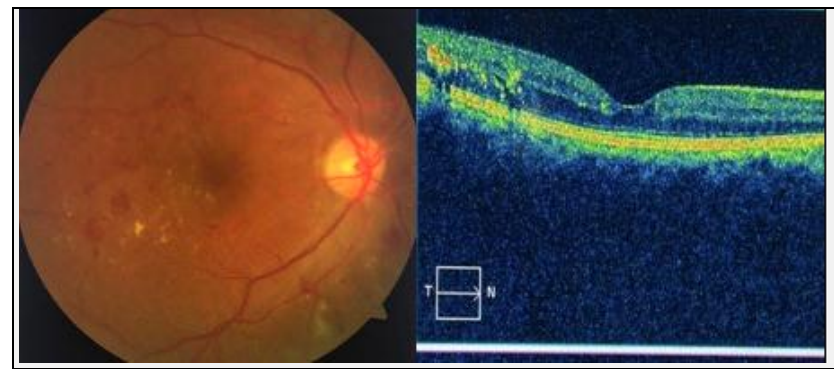

Figure 1. Mild NPDR with CSME with Corresponding OCT Image Showing Diffuse Macular Oedema with Increased Macular Thickness
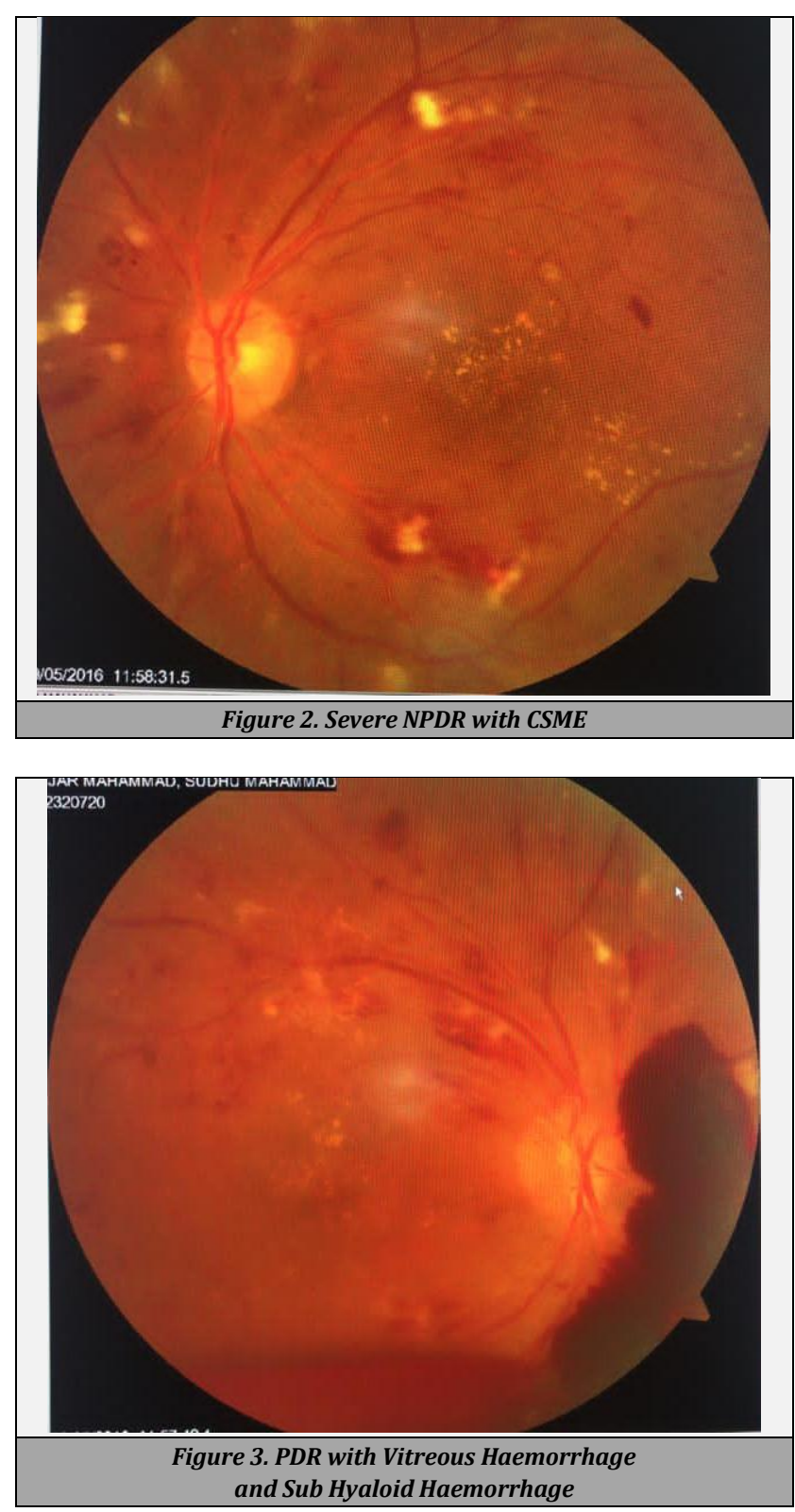

Microalbuminuria correlates well with diabetic retinopathy especially proliferative retinopathy. In a population-based study by Sobngwi et al. in Cameroon they found microalbuminuria to be significantly elevated in patients with advanced retinopathy. ${ }^{26}$ Won Jee lee et al. looked at the relationship between diabetic nephropathy and retinopathy in the Korean population and found that the severity of nephropathy correlated with the severity of retinopathy, ${ }^{27}$ The likelihood of manifesting nephropathy was higher in PDR patients. These findings correlated with the 
findings in our study. Many patients who presented with vitreous haemorrhage or advanced PDR had poorer visual outcomes and had lower baseline GFR rates and more advanced kidney damage. The visual recovery in these patients was poor and the end-organ damage was advanced. Significant visual and systemic morbidities were associated with advanced kidney damage. In a study by Manaivat et al. they divided the population based on mild-moderate and severe retinopathy and correlated the microalbuminuria levels in these three levels and found that the levels were elevated with the progressive increase in severity of diabetic nephropathy. ${ }^{28}$ The results were similar to ours and the conclusions were extrapolatable. At the end of the study, we were able to identify two sets of patients. The first set had only diabetic retinopathy, and the second, with diabetic retinopathy and DN with renal lesions (microalbuminuria).

In the first set, the duration of diabetes mellitus and $\mathrm{HbA} 1 \mathrm{c}$ were the most important risk factors and in the second set, HbA1c levels, renal profile and blood pressure were the most important. The progression of DR with vision-threatening complications and coexisting DN depended on the initial findings on presentation, duration and control of diabetes and was compounded by comorbidities including hypertension, dyslipidaemia, and serum creatinine levels.

\section{CONCLUSIONS}

DR and DN remain an enigmatic topic as there can be overlapping features and variable courses with relevance to both. We found that significant association does exist and that all patients with retinopathy have to be screened for nephropathy and vice versa. This methodical protocol in all patients with DM will prevent vision-threatening complications and life-threatening consequences.

Data sharing statement provided by the authors is available with the full text of this article at jemds.com.

Financial or other competing interests: None.

Disclosure forms provided by the authors are available with the full text of this article at jemds.com.

\section{REFERENCES}

[1] Lee R, Wong TY, Sabanayagam C. Epidemiology of diabetic retinopathy, diabetic macular edema and related vision loss. Eye and vision (London, England) 2015;2:17.

[2] Chawla A, Chawla R, Jaggi S. Microvasular and macrovascular complications in diabetes mellitus: Distinct or continuum? Indian Journal of endocrinology and metabolism 2016;20(4):546-51.

[3] Nathan DM, Genuth S, Lachin J, et al. The effect of intensive treatment of diabetes on the development and progression of long-term complications in insulindependent diabetes mellitus. The Diabetes Control and Complications Trial Research Group. N Engl J Med 1993;329(14):977-86.

[4] Kowalski A, Krikorian A, Lerma EV. Diabetic nephropathy for the primary care provider: new understandings on early detection and treatment. The Ochsner Journal 2014;14(3):369-79.

[5] Klein R, Klein BE, Moss SE, et al. The Wisconsin epidemiologic study of diabetic retinopathy. IV. Diabetic macular edema. Optholmology 1984;91(12):1464-74.

[6] Currie G, Dellee C. Proteinuria and its relation to cardiovascular disease. International Journal of Nephrology and Renovascular Disease 2014;7:13-24.

[7] Cade WT. Diabetes-related microvascular and macrovascular diseases in the physical therapy setting. Physical Therapy 2008;88(11):1322-35.

[8] UK Prospective Diabetes Study (UKPDS) Group. Intensive blood - glucose control with sulphonylureas or insulin compared with conventional treatment and risk of complications in patients with type 2 diabetes (UKPDS 33). Lancet 1998;352(9131):837-53.

[9] Semeraro F, Cancarini A, Rezzola S, et al. Diabetic retinopathy: vascular and inflammatory disease. Journal of Diabetes Research 2015;2015:582060.

[10] Rouf RSB, Ashrafuzzaman SM, Latif ZA. Association of retinopathy with chronic kidney disease in diabetes mellitus. BIRDEM Medical Journal 2018;8(3):210-14.

[11] Molitch ME, Defronzo RA, Franz MJ, et al. Nephropathy in diabetes. American Diabetes Association: Diabetes Care 2004;27(Suppl 1):S79-S83.

[12] Stevens LA, Schmid CH, Zhang YL, et al. Development and validation of GFR - estimating equations using diabetes, transplant and weight. Nephrology Dialysis Transplantation 2010;25(2):449-57.

[13] Early Treatment Diabetic Retinopathy Study Research Group. Classification of diabetic retinopathy from fluorescein angiograms: ETDRS report number 11. Ophthalmology 1991;98(Suppl 5):807-22.

[14] Jamal S, Ali MH, Ayub MH, et al. Frequency and grading of diabetic retinopathy in diabetic end stage renal disease patients. Pakistan Journal of Ophthalmology 2016;32(2):64-9.

[15] Melton LJ, Palumbo PJ, Chu-Pin C. Incidence of diabetes mellitus by clinical type. Diabetes Care 1983;6(1):75-86.

[16] Awad SF, Huangfu P, Ayoub HH, et al. Forecasting the impact of diabetes mellitus on tuberculosis disease incidence and mortality in India. Journal of Global Health 2019;9(2):020415.

[17] Sabanayagam C, Foo VH, Ikram MK, et al. Is chronic kidney disease associated with diabetic retinopathy in Asian adults? J Diabetes 2014;6(6):556-63.

[18] Lee WJ, Sobrin L, Kang MH, et al. Ischemic diabetic retinopathy as a possible prognostic factor for chronic kidney disease progression. Eye 2014;28(9):1119-25.

[19] Poole G. Intercapillary glomerulosclerosis and the Kimmelstiel-Wilson Syndrome. Postgraduate Medical Journal 1953;29(329):137-46.

[20] Kanauchi M, Kawano T, Uyama H, et al. Discordance between retinopathy and nephropathy in type 2 diabetes. Nephron 1998;80(2):171-4.

[21] Biswas G, Dutta S, Mandal S, et al. Association of diabetic maculopathy with diabetic nephropathy - a study. Journal of the Indian Medical Association 2002;100(9):567-8.

[22] Ravid M, Brosh D, Ravid-Safran D, et al. Main risk factors for nephropathy in type 2 diabetes mellitus are plasma 
cholesterol levels, mean blood pressure and hyperglycemia. Archives of Internal Medicine 1998;158(9):998-1004.

[23] Wilkinson-Berka JL, Miller AG. Update on the treatment of diabetic retinopathy. The Scientific World Journal 2008;8:98-120.

[24] Ahmed MH, Elwali ES, Awadalla H, et al. The relationship between diabetic retinopathy and nephropathy in Sudanese adult with diabetes: population based study. Diabetes \& Metabolic Syndrome 2017;11(Suppl 1):S333S6.

[25] Nusinovici S, Sabanayagam C, Teo BW, et al. Vision impairment in CKD patients: epidemiology, mechanisms, differential diagnoses, and prevention. American Journal of Kidney Diseases 2019;73(6):846-57.
[26] Sobngwi E, Mbanya JC, Moukouri EN, et al. Microalbuminuria and retinopathy in a diabetic population of Cameroon. Diabetes Research and Clinical Practice 1999;44(3):191-6.

[27] Lee WJ, Sobrin L, Lee MJ, et al. The relationship between diabetic retinopathy and diabetic nephropathy in a population-based study in Korea (KNHANES V - 2, 3). Investigative Ophthalmology and Visual Science 2014;55(10):6547-53.

[28] Manaviat MR, Afkhami M, Shoja MR. Retinopathy and microalbuminuria in type II diabetic patients. BMC Ophthalmology 2004;4(1):9. 